\title{
In-situ structural evaluation during the fibre deposition process of composite manufacturing
}

\author{
F. Heinecke ${ }^{1}$ and T. Wille ${ }^{2}$
}

\begin{abstract}
Within the European funded project ECOMISE a new approach for composite manufacturing is developed. This approach provides key technologies for industry 4.0 in order to maximize process efficiency at reduced cost and time while maintaining structural requirements. In detail, process simulation methods, online process monitoring systems as well as methods for in-situ structural evaluation and process adjustment in case of process deviations are implemented and linked via databases. This paper describes the new overall concept as well as the specific in-situ structural evaluation approach, exemplarily applied to the fibre deposition process. Prior to manufacturing typical manufacturing features such as locally varying fibre orientation, gaps and overlaps are studied based on given knowledge from previous manufacturing as well as from process simulation. The effect of selected features on the structural properties is investigated for the expected parameter ranges. The real detected features are provided by an online monitoring system during the fibre deposition process. Based on these results an insitu structural evaluation of detected features is performed already during manufacturing in combination with a decision making with respect to required part correction. The developed key technologies and tools for the insitu evaluation process are presented, and their prototype application is shown during manufacturing of an aeronautic wing cover demonstrator.
\end{abstract}

Keywords: In-situ structural evaluation, effects-of-defects, multi-scale analysis, surrogate modelling, gap, overlap, automated fibre placement (AFP)

\section{Introduction}

Contemporary composite part development and manufacturing, in particular of high performance light weight structures, is still requiring a high effort in order to find optimal process parameters and to meet required qualities and tolerances. In case of fibre deposition the processes of pick and place, tape placement or fibre placement are industrially applied enabling a high degree of automation, while meeting high structural requirements. However, depending on the complexity of the part as well as on the selected process technology and material type the resulting semi-finished products still contain different manufacturing deviations that are to be considered. Already in case of simple fibre deposition onto flat surfaces tolerances of fibre orientations, ply contours or ply thickness as well as gaps and overlaps appear [1]. In case of more complex shaped geometries or fibre steering tapered gaps and overlaps cannot be avoided, and process difficulties lead to fibre waviness, wrinkles or twisted tows [2]. In order to balance these tolerances and to avoid defects during fibre deposition optimum process parameters are usually determined for each specific application taking into account prior defined structural requirements. Depending on the complexity of the part this requires a high effort, but certain risks for manufacturing defects still remain. Furthermore, structural requirements are often derived from simplified and conservative rules, while neglecting possible reserves for individual applications. This conservatism comprises two drawbacks: On the one hand structural reserves are often not exploited. On the other hand non-added value manufacturing and rework processes unnecessarily increase manufacturing costs [3].

In order to improve the process robustness isolated monitoring systems are already applied to monitor process parameters and to provide information for process adjustments. Yet, these adjustments are performed against prior defined conservative tolerances of respective process parameters. No information is provided on the actual

\footnotetext{
${ }^{1}$ German Aerospace Center, Institute of Composite Structures and Adaptive Systems, Lilienthalplatz 7, 38108, Braunschweig, Germany, falk.heinecke@dlr.de, +49(0)531 295 2312, ORCID: orcid.org/0000-0002-9299-4078

${ }^{2}$ German Aerospace Center, Institute of Composite Structures and Adaptive Systems, Lilienthalplatz 7, 38108, Braunschweig, Germany, tobias.wille@dlr.de, $+49(0) 5312953012$
} 
material properties affected by process deviations. Moreover, no evaluation is performed whether a certain knock-down of material properties is significantly affecting the structural performance of a part, especially in case of a defect located in minor loaded areas of the part.

Aiming at significant reduction of manufacturing costs, rework and part rejections a new approach is presented within this paper. This approach provides an in-situ structural evaluation of part deviations occurring during composite manufacturing, exemplarily shown for the fibre deposition process. By integrating an in-situ evaluation an early feedback is given, allowing for decision making whether to allow or correct current defects before handing over to the next manufacturing step. In order to enable this throughout approach following key technologies are to be provided:

- Online monitoring systems must be capable to measure relevant part and process parameters and also to characterize defects with required reliability (i.e. capability to measure and to detect deviations within specified tolerances without fault) in real-time.

- Profound physical understanding and efficient analysis tools are required for real-time evaluation of the actual effects of defects, i.e. to assess the influence of certain defects on the structural behaviour.

Within the following section 2 the overall evaluation process is explained by providing information on required methods and tools as well as related input and data mining. For this workflow particular attention is payed to the integration of highly efficient methods with required accuracy in order to meet real-time evaluation capability. Subsequent section 3 is dedicated to the developed methods required for the evaluation of defects on the local defect level. The focus is laid upon the analysis of fibre deviations during the automated fibre placement process (AFP). Section 4 describes the procedure for evaluating the resulting structural performance taking into account these local deviations. The procedure is demonstrated by means of an aeronautic wing cover manufactured by AFP. Section 5 concludes the results and gives an outlook for further research suggested.

\section{Evaluation Procedure}

Current process chains of composite part production already include several quality assurance checks. Incoming goods (e.g. fibre and resin material) are checked with respect to their specifications. During manufacturing sensors are increasingly applied to capture process parameters such as time-dependent pressure or temperature. Traveller coupons are additionally used for subsequent material analysis, and NDT techniques are applied directly after manufacturing in order to check e.g. for fibre waviness, pores, delamination or geometrical deviations. Baseline for all of these quality checks are prior defined global tolerances for a component of a certain material. Yet, no distinction is made between differentially loaded areas. Also quality checks during manufacturing are only referred to process parameters but not directly to determining structural properties. Moreover, current evaluation methods are involving significant manual effort and thus, hindering an automated and direct feedback and early decision making on acceptance, adjustments or rejection.

The novel evaluation procedure enables an early and fast evaluation of manufacturing deviations with respect to their effect on the structural behaviour on component level. Special care is taken to the analysis of the effects of process deviations on the resulting structural mechanical properties. The development and integration of highly efficient methods for so-called in-situ assessment need to consider different types of deviations directly during manufacturing. Such deviations are classified in two categories:

- Defects, which can be directly considered within the global structural analysis (e.g. fibre angle deviations, thickness deviations)

- Defects, which have to be analysed using detailed local models in advance to the global structural analysis (e.g. gaps, overlaps, undulations).

A concept was developed in order to account for these two categories. For the first type of defects all measured deviations are directly transferred via an appropriate mapping method onto the global structural model for the Insitu Structural Evaluation, which corresponds to the third step of the developed concept (cf. Fig. 1). 
Yet, the second type of defect usually affects not only a single ply but the overall laminate and therefore requires additional analysis effort on defect level. The overall procedure to evaluate the effects of manufacturing deviations is depicted in Fig. 1.

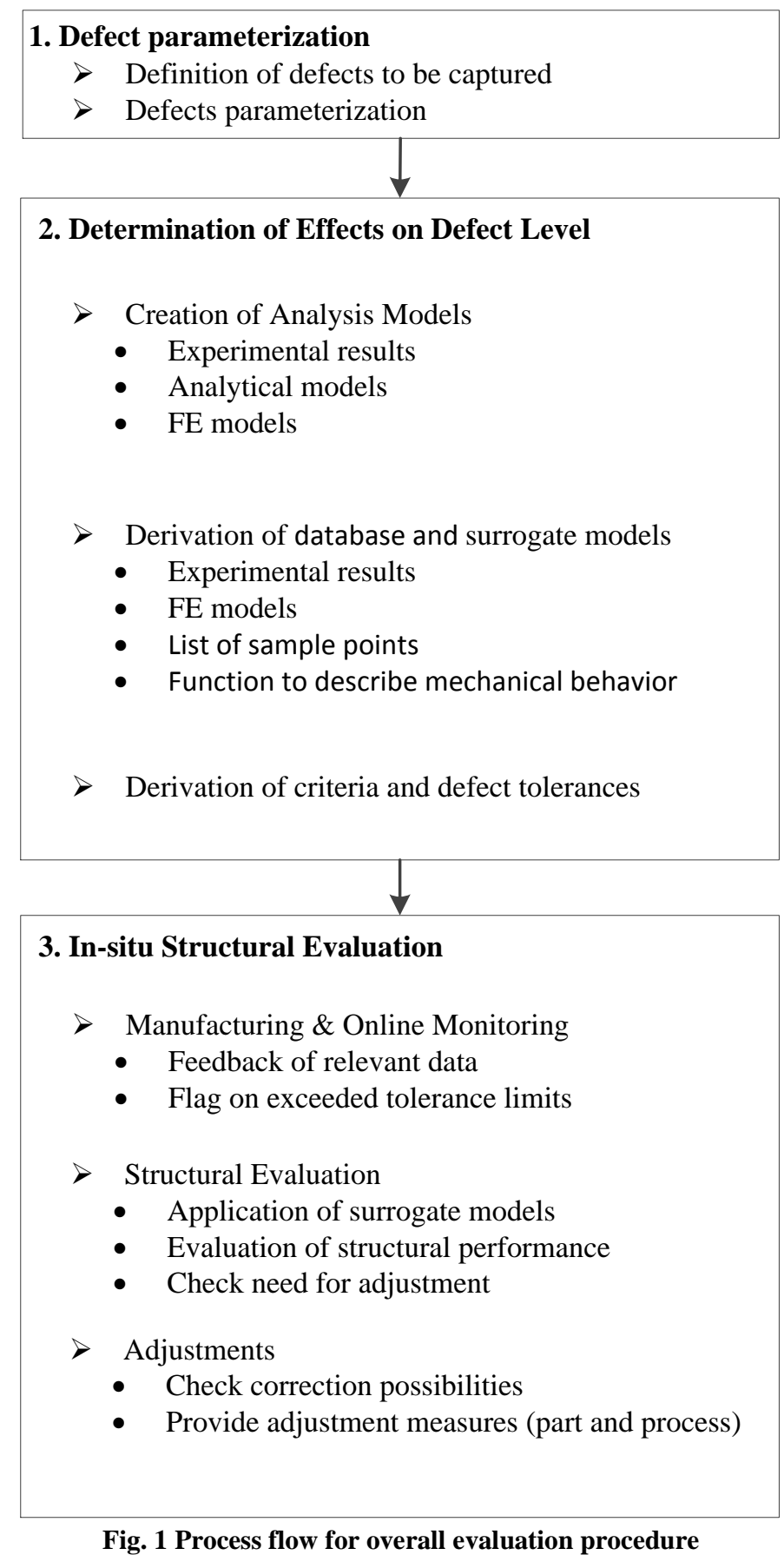

In the first step the Defect Parameterization takes place for the relevant defects, which are to be considered within the in-situ evaluation. This step, which also provides a defect parametrisation, is performed already in the design phase, mostly experience and knowledge based, respectively.

The second step is dedicated to the Determination of Effects on Defect Level. This step is also to be performed in advance to the actual manufacturing and establishes the in-situ capabilities for the overall manufacturing system. Here, a database is created, which is based on experimental and/or virtual (based on detailed FE models) material tests of laminates containing representative generic imperfections. This so-called Effects-of-Defects database (short EoD database) contains knock-down factors for the stiffness and strength properties of each individual layer within a laminate. 
During the actual manufacturing, in the third step (In-situ Structural Evaluation), a comparison of online measurement data with the EoD database takes place. This approach enables an efficient evaluation at the component level and an instant derivation of potential corrective measures. For this purpose an online fibre measurement system provides fibre angle, fibre undulations, ply thickness, gaps and overlaps. The observed parameters are correlated to the corresponding knock-down factors, whereby the change of failure behaviour and load redistribution due to imperfections can be represented. Surrogate models based on the EoD database enable an efficient determination of these knock-down factors during the actual manufacturing process.

Within the following sections 3 and 4 the developed methods are described for determining the effects of defects on local defect level (step 2) and performing the in-situ structural assessment on component level (step 3).

\section{Determination of Effects on Defect Level}

Current state-of-the-art software tools already provide methods such as sub-modelling or multi-scale analysis to take into account local particularities on a structural level [4]. These methods are applicable within the detailed structural design phase in order to evaluate certain structural particularities. They can also be used during the concession phase after manufacturing in order to assess specific part deviations that have been identified by NDT. Yet, these analysis methods still require a comparably high computational effort and therefore cannot be applied for real-time analysis and evaluation during manufacturing. Also most analysis procedures are applied to single plies and therefore don't capture the knock-down effect on adjacent plies due to local load redistribution $[5]$.

An enhanced multi-scale analysis technique is presented that considers local material discontinuities on laminate level. Manufacturing induced defects with out-of-plane characteristics are basically not captured by shell-based models, since just the laminate mid-plane is modelled. Utilization of 3D models of defective laminates enables to consider the $3 \mathrm{D}$ stress state when investigating the effect of the defect

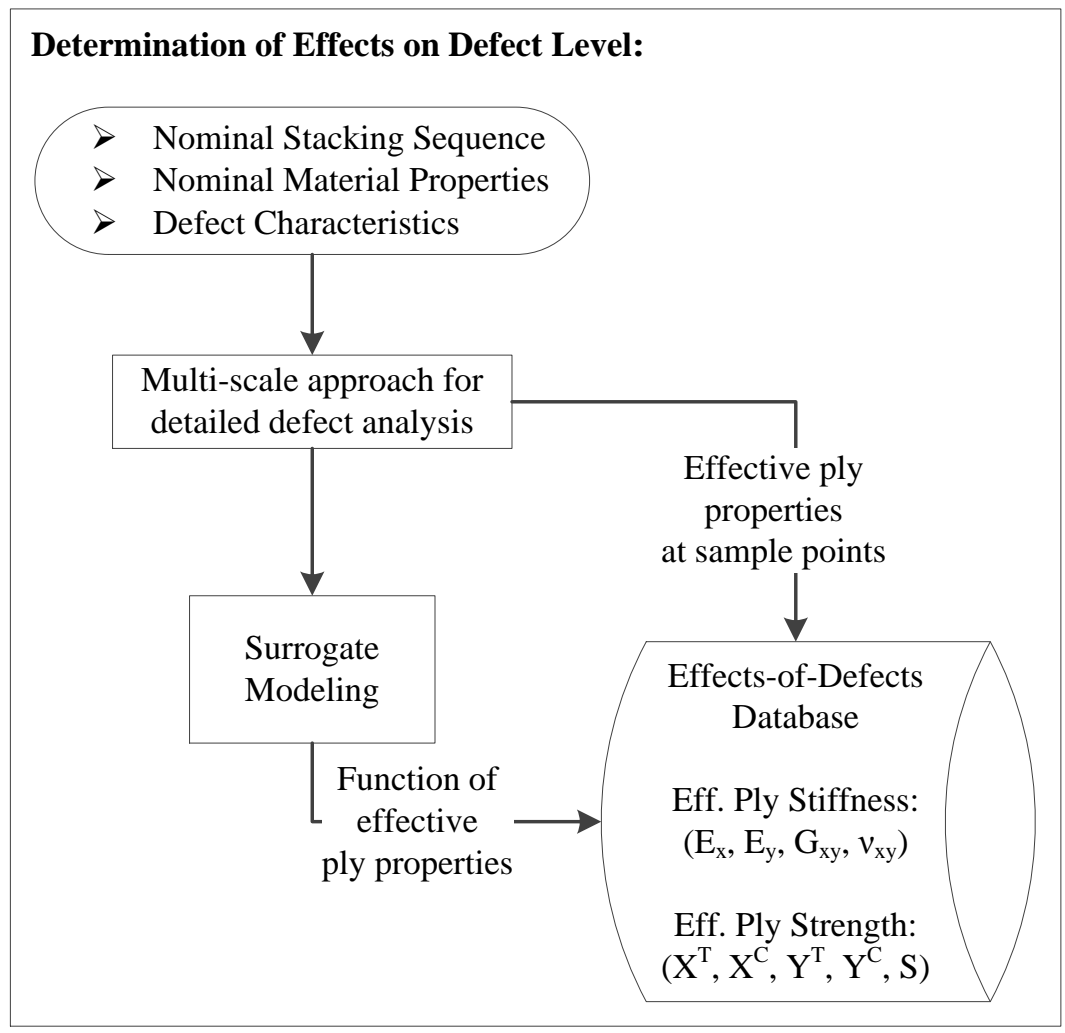

Fig. 2 Process flow for determining effects on defect level

on the material behaviour. A suitable macroscopic resolution of certain material particularities (e.g. gaps or undulations) is provided by homogenization of material properties using parameterized and detailed 3D FE 
models. On the basis of a specific defect parameterization effective material properties are calculated and locally assigned. This allows determining the as-built part properties for certain deviations of the fibre architecture within the laminate.

In order to provide real-time simulation capability during manufacturing the EoD database as well as surrogate models are to be generated in advance to the actual manufacturing, cf. process flow in Fig. 2. Based on given input regarding stacking sequence, material properties and defect characteristics detailed parametric defect models are generated and used to determine the effective ply stiffness and strength values for predefined sample points. On the one hand these results are stored within the EoD database for direct use. On the other hand they are further exploited for deriving surrogate models of ply stiffness and strength to enhance the EoD database.

\subsection{Multi-scale Approach}

In general a multi-scale analysis is composed of different steps. The process starts with the generation of a local FE model, mostly called representative volume element (RVE). In this paper these local FE models are called super-elements, since material periodicity is non-existent. Fibre architecture and defect particularities are typically provided as explicit information from measurement or as generic information based on a specific parameterization. Subsequently, all information is consolidated into the super-element of the defect area. Finally, virtual tests are performed in order to determine required effective material stiffness and strength parameters [6]. These material parameters can be calculated by means of conducting a homogenization approach. Such homogenization can be performed empirically, analytically by using rules of mixture or numerically. The approach presented e.g. by [7] considers the effect of localized fibre waviness in unidirectional composites but is also applicable for other types of defects, such as gaps and resulting 3D fibre undulation.

The effect of a defect on the mechanical behaviour of a local laminate depends on the respective layup and material properties [8]. Particularly, the effect of 3D effects on the material properties is of importance and requires 3D super-element models. In order to provide a sufficient database different super-elements have to be created for all types of relevant manufacturing defects. For this work parameterized super-element models are automatically generated and analysed using a Python based framework [9]. The defective laminate is appropriately modelled using either available measurement data or generic geometry parameters. In case of explicitly available defect data from measurement direct modelling can be performed, e.g. by importing measurement data and using advanced meshing techniques. Considering generic investigations an efficient parametrization is required for defect type, shape and size.

According to the individual manifestations of the manufacturing defects, such as gaps, overlaps or undulations, different generic geometry parameters are necessary, which represent the respective defect characteristics properly. In this context the challenge is to keep the number of parameters small enough to achieve an acceptable computational effort for deriving surrogate models, but maintain the required representation. Garnich and Karamai [7] and Croft et al. [10] revealed that most of the relevant defects can be represented in good approximation by two parameters maximum. In case of in-plane and out-of-plane fibre undulations the amplitude and the wavelength is commonly used to represent the geometric characteristic. As described by Croft et al. [10] the geometric characteristics of gaps and overlaps can be captured in the same manner. Hence, an analogous set of characteristic geometric parameters is chosen for the gap and overlap defect.

Using the information about the nominal layup, the nominal material properties for each ply as well as the defect characteristic parameters a detailed 3D super-element model is created. Exemplarily, Fig. 3 right depictsa 3D FE model of a defective laminate with a missing tow in the 12th ply, which leads to a gap within the ply as well as to out-of-plane undulation in subsequent plies. The super-element is composed of 3D continuum elements, where each element row represents one ply and each colour represents a different material. 
The evaluation of results is the last step performed during the multi-scale analysis. An approach proposed by Kärger and Kling [11] is adapted for this purpose (refer also to [12]). In order to get the baseline, to compare against, the effective material properties of the pristine laminate (refer to Fig. 3 left) are evaluated once per layup. This calculation is reiterated for each individual defect occurring within the respective layup. Within this step the actual analysis of the local FE model and the homogenisation is conducted. To determine the effective material properties different types of boundary conditions can be used [13]. In this work periodic uniaxial displacement boundary conditions are applied to the super-element [14].

Since the gap affected domain has pure resin properties (refer to Fig. 3 right dark coloured domain in first undulated ply), which are considerably smaller than the surrounding stiffness, this region attracts less stress. Stress concentration occurs at the edges of the gap due to a stress re-distribution, which finally changes the laminae properties compared to the pristine laminate. The effective stiffness can be computed by evaluating the individual stress components averaged for all elements of the super-element. The difference of material properties is expressed layer-wise as so-called knock-down factors. The knock-down factor determines the effect of the respective defect as the percentage of the nominal stiffness value (refer to Fig. 4) and the nominal strength value (refer to Fig. 5), respectively. For retrieving the effective strength the Tsai-Wu failure criterion is used.

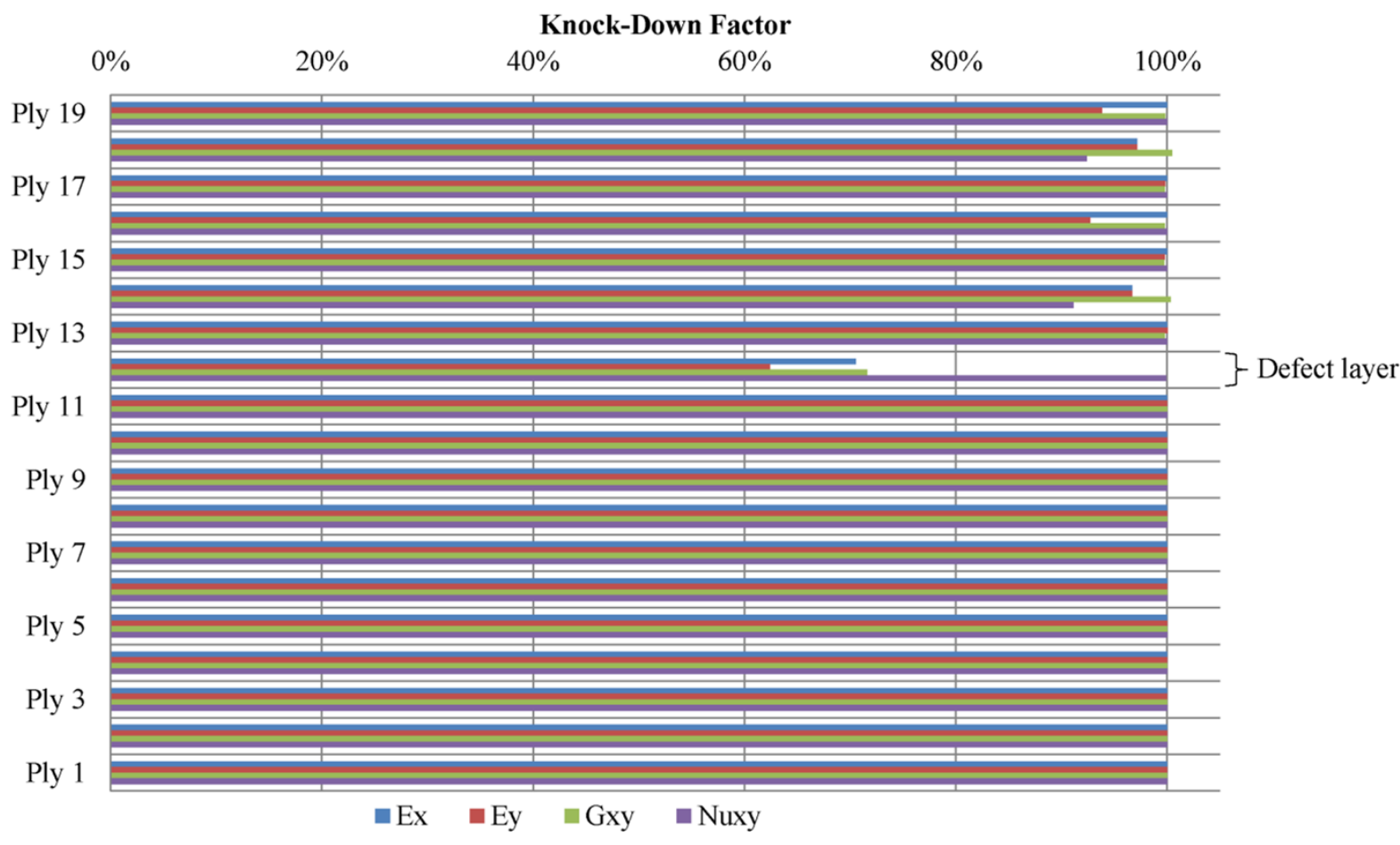

Fig. 4 Knock-Down Factors of the nominal stiffness material properties 
For the subsequent structural evaluation and (re-)qualification only the Young's modulus in fibre direction $\left(\mathrm{E}_{\mathrm{x}}\right)$, the Young's modulus in transverse direction $\left(\mathrm{E}_{\mathrm{y}}\right)$, the in-plane shear modulus $\left(\mathrm{G}_{\mathrm{xy}}\right)$ and the in-plane Poisson's ratio $\left(v_{\mathrm{xy}}\right)$ is needed. The same applies to the strength properties. Just the tensile and compressive strength in fibre direction $\left(\mathrm{X}^{\mathrm{T}}, \mathrm{X}^{\mathrm{C}}\right)$, in transverse direction $\left(\mathrm{Y}^{\mathrm{T}}, \mathrm{Y}^{\mathrm{C}}\right)$ and the shear strength $(\mathrm{S})$ are important. By simulating the same layup with various defect configurations a set of sample points is generated, which is in turn used for the subsequent surrogate modelling.

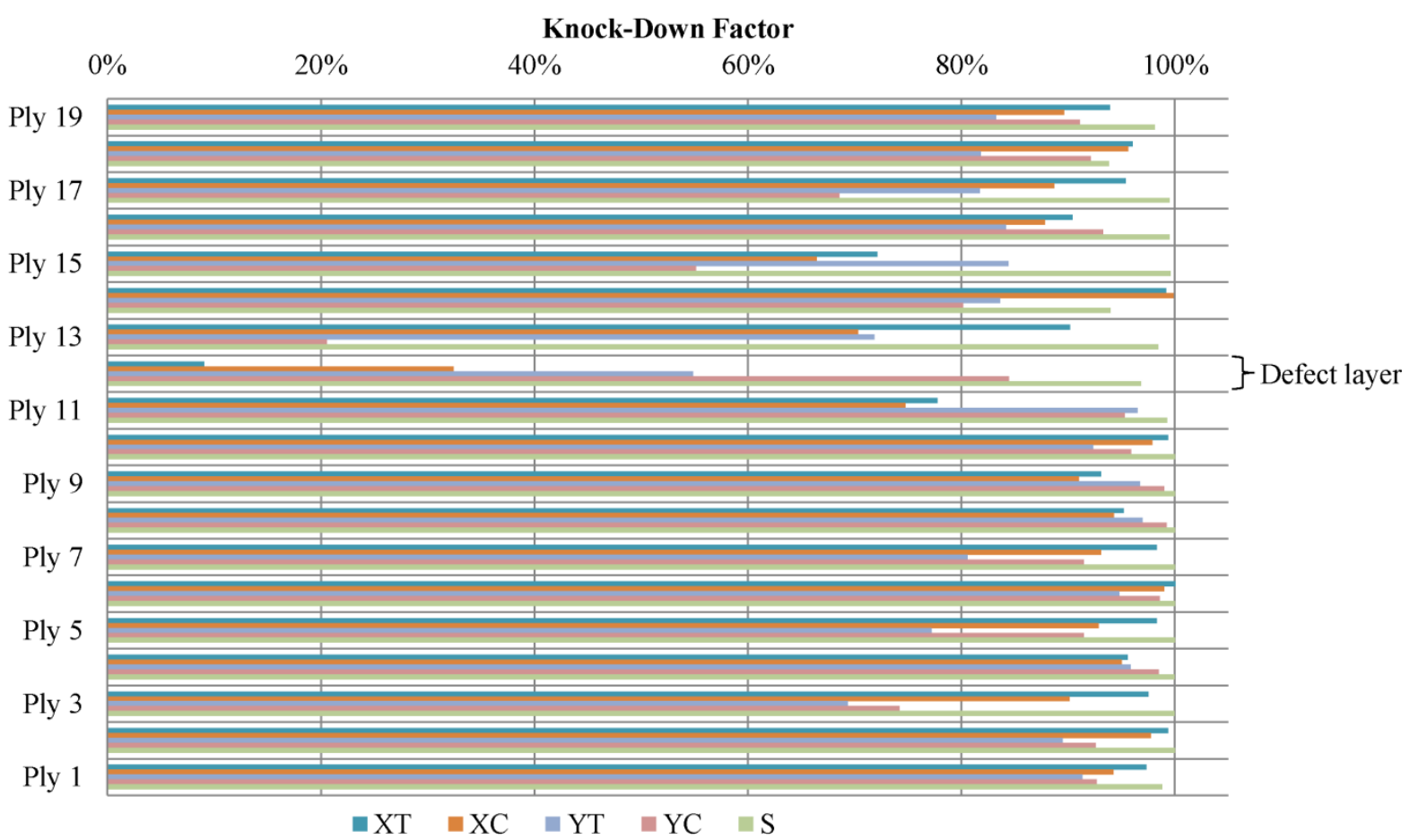

Fig. 5 Knock-Down Factors of the nominal strength material properties

\subsection{Surrogate Modeling}

Serving the overall requirement for real-time assessment the potential of surrogate models is exploited. For this purpose individual surrogate models are derived for each knock-down factor in order to evaluate the effects of defects during the actual manufacturing phase.

The principle workflow makes use of the OpenTurns [15] open source framework in conjunction with a Python [9] implementation. This framework comprises several so-called designs of experiments (DoE) to create appropriate sample points for subsequent surrogate modelling. The Latin Hypercube Sampling technique is widely used for engineering problems, as discussed e.g. by Forrester et al. [16]. In terms of computational effort it has also been proven to be suitable for this work to create an adequate DoE for the required parameter space.

Regarding the subsequent step of surrogate modelling several techniques are available to solve engineering problems, cf. [17] and [18]. The most common surrogate modelling techniques are the Polynomial Regression, the Radial Basis Functions, the Kriging and the Support Vector Regression. Due to their different approaches for creating a surrogate model they also differ in accuracy, robustness and suitability [19]. Because of the good relation between efficiency and accuracy the Kriging algorithm is chosen to generate the surrogate model interpolating the effects of defects on the local level. The parameter space of these models on defect level consists of the affected defective layer (first parameter) and the width of the defect (second parameter).

Fig. 6 exemplarily depicts the visualization of a resulting surrogate model for the knock-down factor of the laminate property $\mathrm{E}_{\mathrm{y}}$ (i.e. the Young's modulus of the laminate in y-direction) caused by a Gap defect. Depending on the Gap location, described by the position of the defect layer within the layup, and the Gap width the knock-down factor for $\mathrm{E}_{\mathrm{y}}$ varies between 0.68 and 1.0. 


\section{In-Situ Structural Evaluation}

Depending on the geometrical complexity of the part as well as the selected manufacturing technology and composite material different types of deviations can occur. In case of fibre deposition by AFP variations in fibre orientation, ply thickness, gaps, overlaps as well as undulations or folds are relevant imperfections. Within this paper the in-situ structural evaluation process is presented as actual performed during dry fibre placement at the facility of the Netherlands Aerospace Center (NLR), depicted in Fig. 7. A customized laser scanning system from Loop Technologies is mounted directly onto the AFP head measuring thickness profiles and identifying deviations of fibre architecture. Characteristic parameters are directly provided as input for the in-situ structural evaluation process. Within this paper the focus is on the analysis of detected gaps.

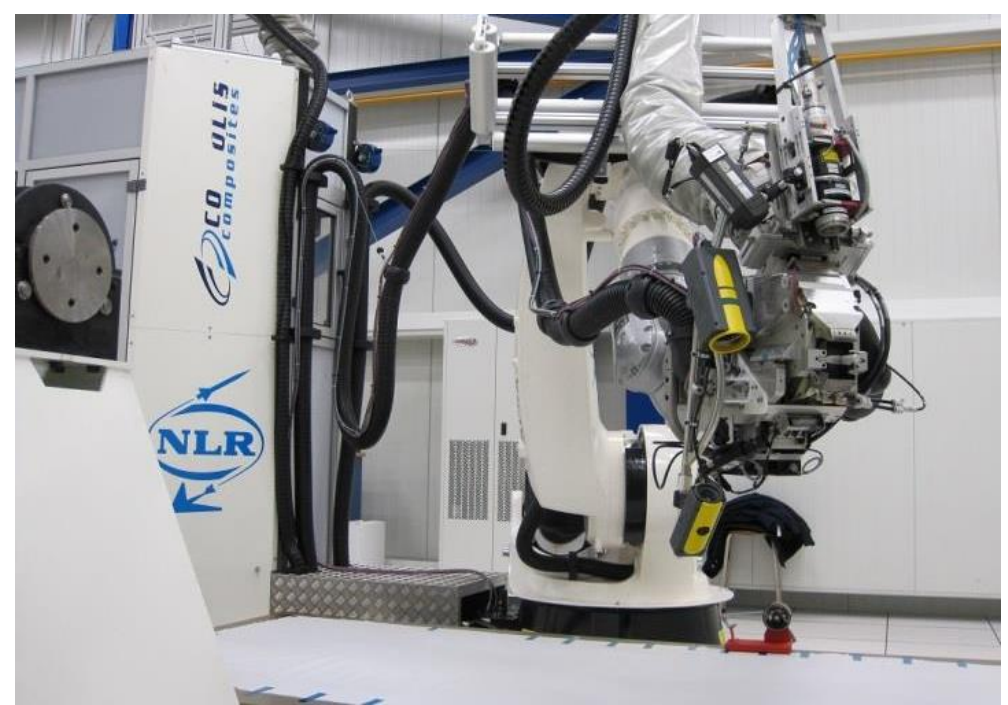

Fig. 7 AFP robot at NLR with integrated 3D optical measurement system

The principle methodology enabling the in-situ structural evaluation is provided in Fig. 8. During the actual manufacturing the as-built model is used for re-evaluation and re-qualification purposes. It is continuously updated with new manufacturing data coming from the online monitoring system. A particular focus is put on the assessment of changes in the structural response in terms of global deformation and failure behaviour. The 
as-built structure is compared to the ideal as-design structure, which is the baseline. Consequently, a decision is made whether the structural quality of the actual component is still acceptable or not.

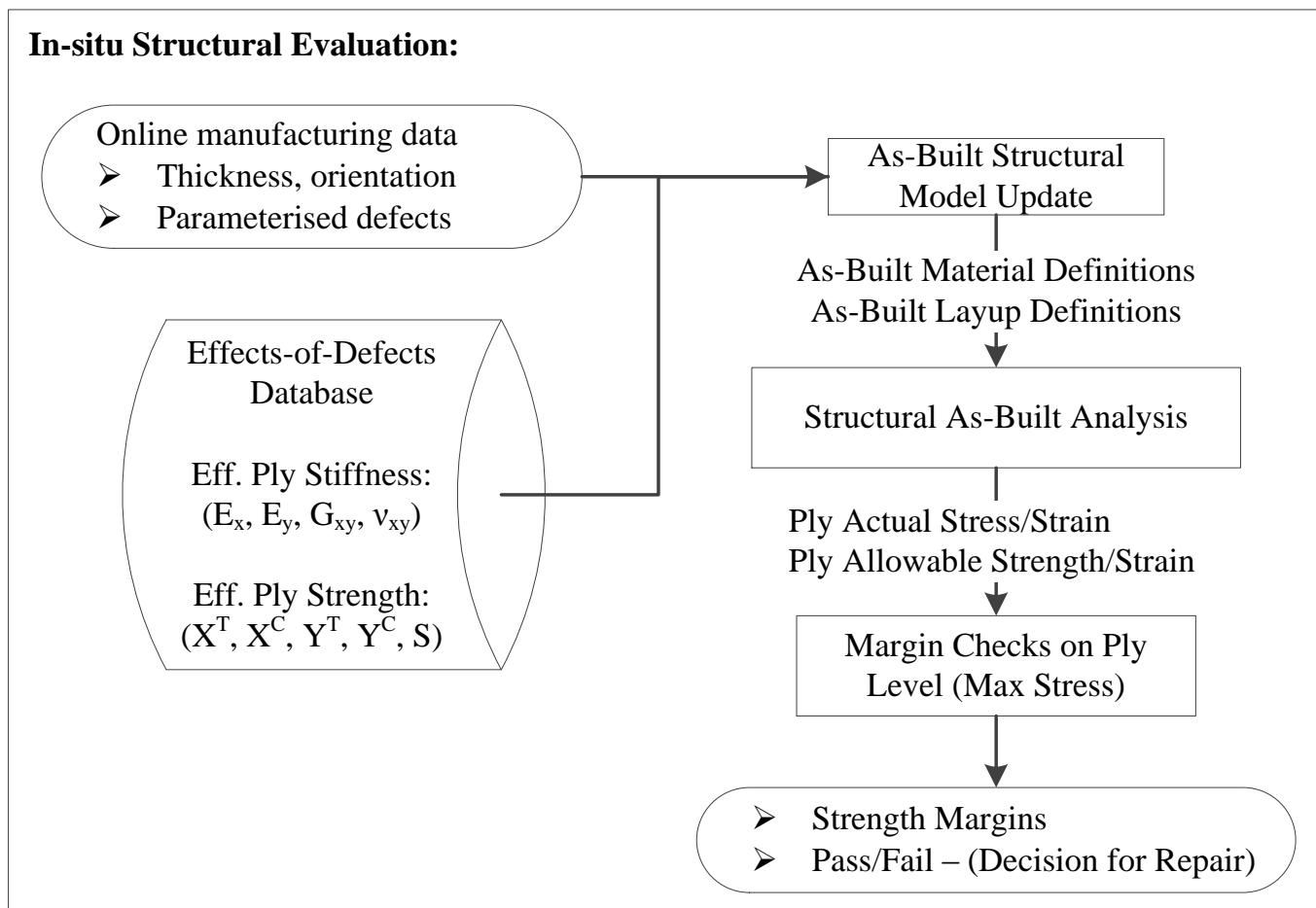

Fig. 8 Process flow - In-situ Structural Evaluation

Following, the in-situ structural evaluation is demonstrated by means of a wing cover of an aircraft manufactured by AFP technology. In Fig. 9 the as-design FE model of the wing cover is illustrated. The part is composed of generic varying layups, which are assigned to different regions of the wing skin as indicated by individual colours. The layup depicted in Fig. 3 left corresponds to the brown coloured domain of the wing skin. The baseline structure, which serves as reference, is assumed to have neither material inhomogeneity nor deviating fibre architecture. It is evaluated with respect to structural allowable (i.e. stiffness, strengths). Starting point is a global structural check. Before manufacturing a stress analysis is available for the idealized preliminary sized model of the wing skin providing expected margins of safety without any defects.

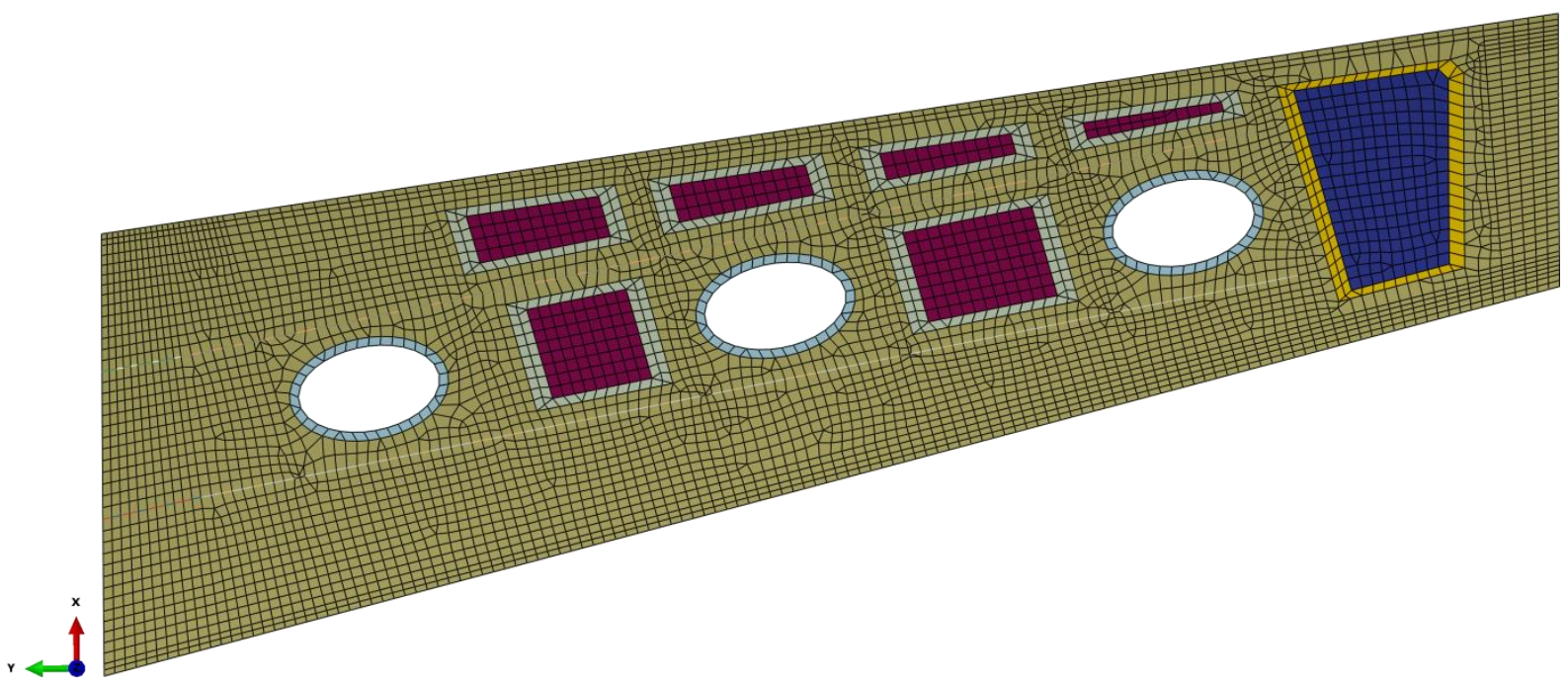

Fig. 9 Idealized preliminary sized model of the wing skin - colours indicating varying layup definitions 
In Fig. 10 the structural response for a representative load case is depicted. For this example the region of the wing cover near to the wing root is exposed to tensile loading. With increasing distance from the wing root the loading condition is more compression dominated.
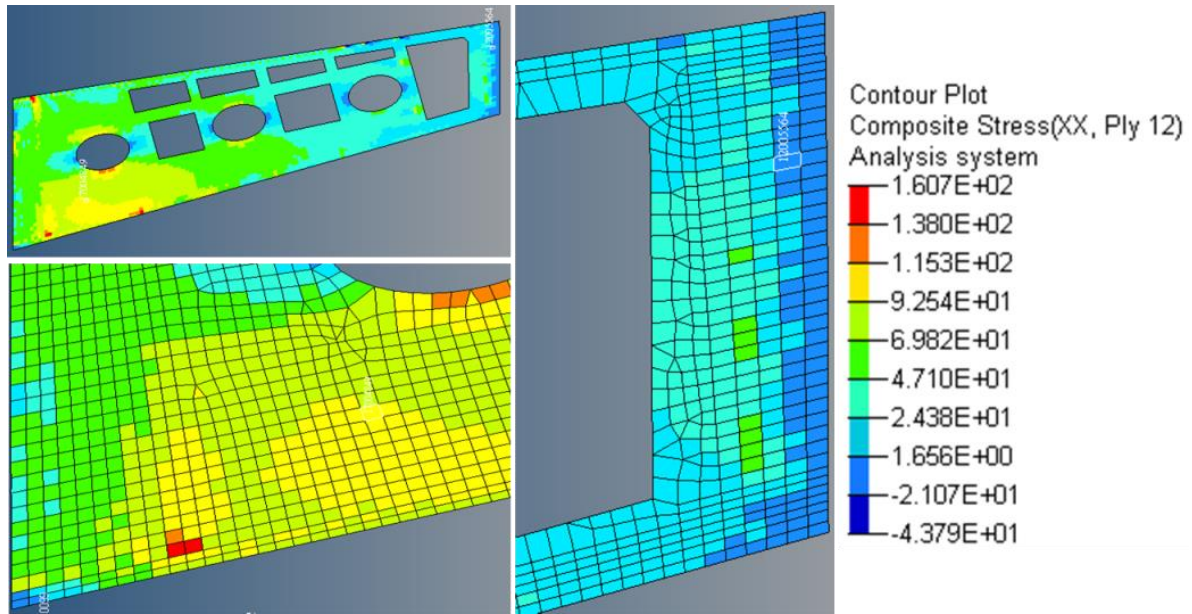

Fig. 10 Baseline Global Finite Element Model (GFEM - on the structural level) check - Tension dominated (lower left) and compression dominated (right) ply stresses in ply 12

Within this paper Equation 1 is used for the calculation of the margins of safety (MoS):

$$
\text { Margin of Safety }=\frac{\text { Allowable Stress }}{\text { Applied Stress }}-1 .
$$

Failure indices serve as basis to determine the MoS. They are calculated assuming the 'Maximum Stress' failure criterion and first ply failure as catastrophic event.

A missing tow defect, which complies with a $6.35 \mathrm{~mm}$ wide gap within the layup, is introduced on purpose to demonstrate the in-situ capabilities of fibre monitoring and structural evaluation. The defect location corresponds to the tensile stressed domain in the wing skin (cf. Fig. 10). Fig. 11 shows a superposition of measured manufacturing imperfections (i.e. gaps and overlaps) and the finite element mesh of the wing skin. Due to the sensitivity of the measurement system a large number of smaller gap and overlap features are detected. However, the missing tow is identified and the characteristic defect parameters are determined by the measurement system. Here the length and the width of a defect are always related to the actual fibre orientation, i.e. the length of the defect corresponds to the fibre direction and the width of the defect to the transverse direction.

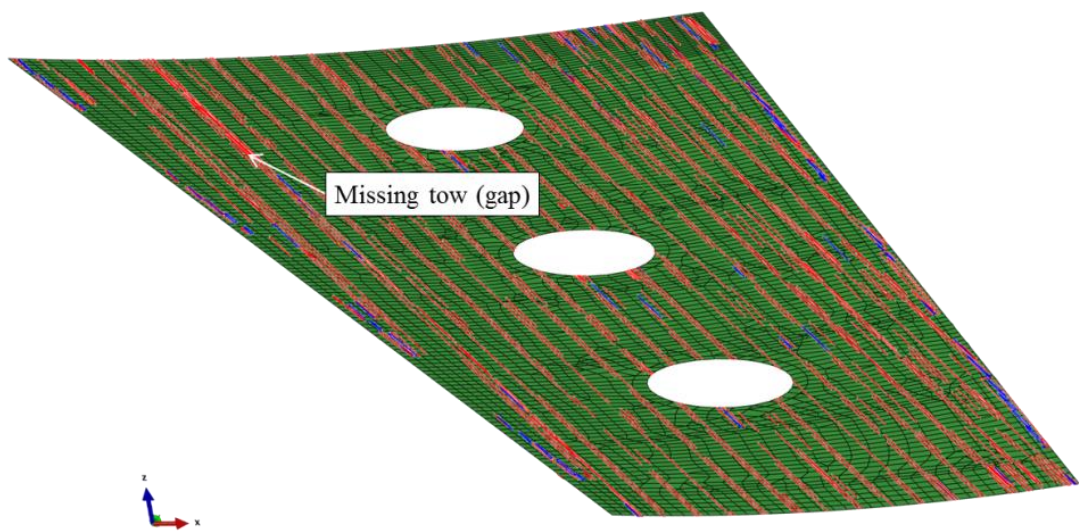

Fig. 11 Gap (red) and overlap (blue) on wing cover detected by 3D measurement system

In the next step the respective structural characteristics of the as-built model are compared with the nominal (baseline) structural specifications. In order to update the actual stress/ deformation state or the actual local material properties of the simulation model feedback and mapping methods are applied in real-time including an update of the corresponding material properties of all finite elements affected by the defect. The following 
changes are introduced to the baseline Global Finite Element Model (GFEM - on the structural level) to account for the detected $6.35 \mathrm{~mm}$ wide gap caused by a dropped tow at ply 12 (refer to Fig. 3 right):

- For each ply additional material ply level property entries are created. Based on the effective ply strength and stiffness properties calculated from the Detailed Finite Element Model (DFEM - on the defect level, refer to section 3).

- For each finite element referencing the newly created effective material properties an additional composite section property entry is created.

- Within the new section property entries, an effective ply thickness is calculated for the defective ply (in this case ply 12) to account the gap.

- $\quad$ The new section properties are assigned to the elements enclosed by the gap area.

The knock-down factors for stiffness and strengths properties of the respective defective layup are provided in Fig. 4 and Fig. 5, respectively. The effective ply thickness of an affected finite element is calculated as follows:

Baseline Ply Cross Section Area: $\quad \mathrm{A}_{\mathrm{Bl}}=$ [Element Width] $*$ [Ply Thickness $]$

Gap Cross Section Area:

$$
\mathrm{A}_{\mathrm{Gap}}=[\text { Gap Width }] *[\text { Ply Thickness }] \text {, }
$$

Effective Ply Thickness:

$$
\mathrm{t}_{\mathrm{eff}}=\frac{\left[\mathrm{A}_{\mathrm{BI}}-\mathrm{A}_{\mathrm{Gap}}\right]}{[\text { Element Width }]}
$$

It should be noted that this approach smears the effect of the local gap across a wider area than the $6.35 \mathrm{~mm}$ gap due to the underlying GFEM with coarse element sizes. By over representing the size of the defect, this approach builds in a degree of conservatism when assessing the effects of the defect on strength properties.

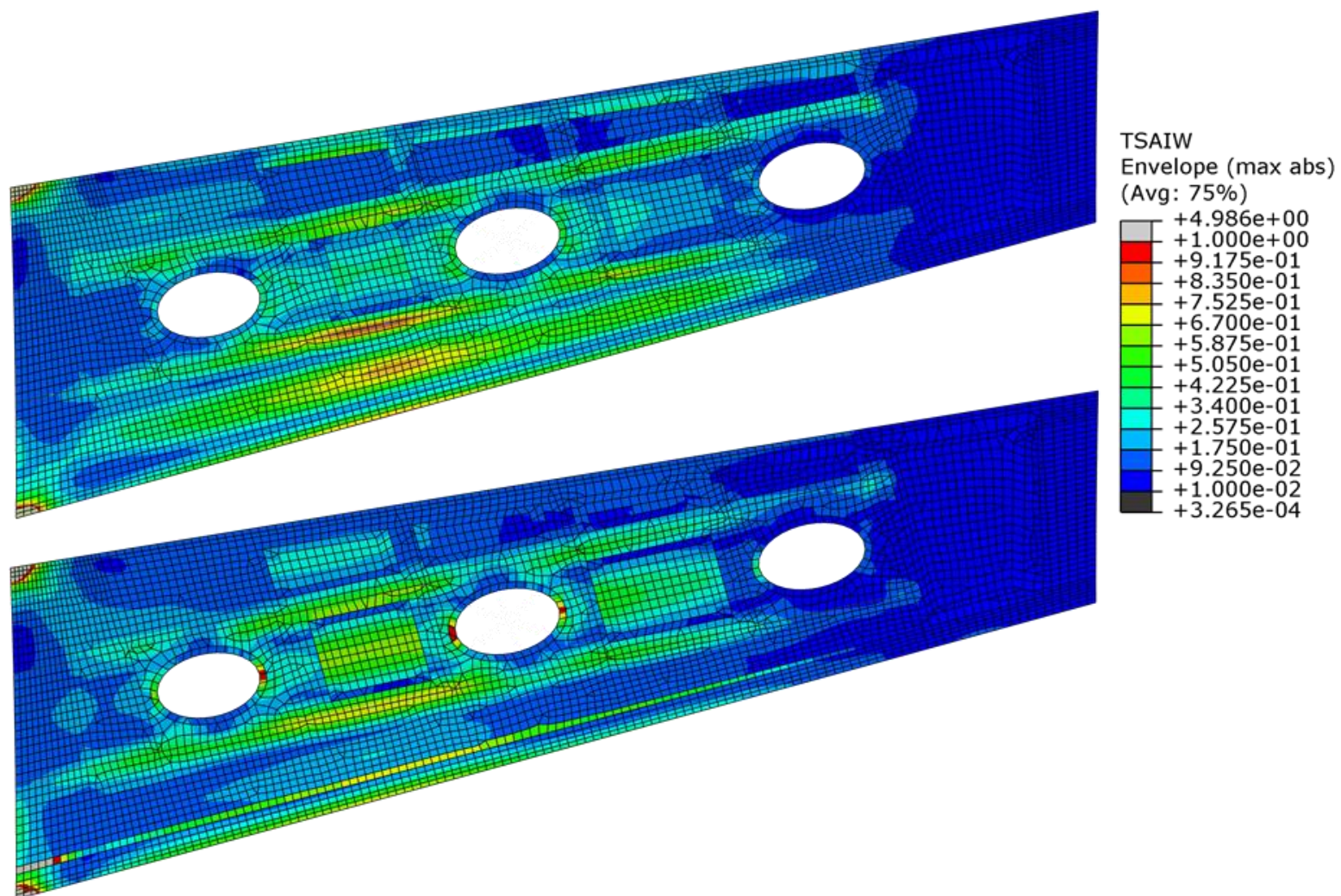

Fig. 12 Material effort envelop according to Tsai-Wu between baseline GFEM (top) and as-built GFEM incl. defect (bottom)

The global structural check is repeated on the as-built wing skin to evaluate the MoS under consideration of defects. The stress analysis is performed applying the same load case but effective material properties for affected elements. Within the global structural check the effects of stress redistribution to regions of pristine 
material are examined. In Fig. 12 material effort is compared between the baseline GFEM and as-built GFEM.

\section{Conclusion and Outlook}

Within the paper a new overall concept and underlying methods for an efficient in-situ evaluation of manufacturing defects already during AFP manufacturing are described. In detail a step-wise approach is introduced comprising detailed preparation analysis and efficient online analysis in order to provide required insitu evaluation capability. Procedures are described for appropriate defect characterisation by means of gaps. Multi-scale analysis methods are described for determining local knock-down factors for all plies of an affected laminate taking into account local load redistribution. Additionally, surrogate modelling techniques are exploited and integrated into a framework. As a result an effects-of-defects database is generated capturing the effects of gaps on local laminate level in form of discrete values or functions.

The in-situ evaluation methodology is demonstrated by assessing the actual effect of gaps on the structural performance of an aeronautical application.It is pointed out how actual online measurement data of the fibre deposition can be used to evaluate occurring manufacturing deviations already during production. The procedure is demonstrated for an industrial aeronautic wing cover application. To this the global impact of local manufacturing imperfections is assessed. On the one hand significant load redistribution can be observed leading to higher material effort in defect-free areas of the part. On the other hand all checked MoS remain acceptable for given load case and defect, thus enabling to continue manufacturing without costly rework. Therefore this paper describes a key technology to enhance future manufacturing and concession processes by automated defect 
assessment and decision making with respect to required process or part corrections. High benefits are particularly expected when applied to early manufacturing steps in order to adjust before provoking further unnecessary costs within subsequent manufacturing steps.

In order to ensure full industrial capability further research and technical developments are required. Experimental testing is recommended for increased understanding of physical behaviour of interacting defects and validation on structural level. Also further demand is identified for efficient and reliable monitoring systems capturing all relevant defects occuring during manufacturing. Moreover, additional effort is required to provide closed-loop automated feedback to the manufacturing facility in case of required process and part correction. There are still unresolved challenges on the way to a reliable correction process. To name just a few aspects: From a technological perspective, fast and robust algorithms are needed, which are capable to create new fibre trajectories and machine paths "on-the-fly", while considering all manufacturing constraints. From the perspective of structure mechanics methods providing the same efficiency have to be developed. To react on structural relevant deviations requires a re-design of the layups. Finding solutions within the multi-dimensional design space is ambiguous. Consequently, such correction sub-process as part of the in-situ structural evaluation is still work in progress.

\section{Acknowledgements}

This work was supported by the European Commission FP7 Programme under Grant NMP2-SL-2013-608667 within the ECOMISE project (Enabling Next Generation Composite Manufacturing by In-Situ Structural Evaluation and Process Adjustment). Collaboration with the Ecomise partners is gratefully acknowledged.

\section{References}

[1] R. Lichtinger, J. Hinterhoelzl, U. Beier and K. Drechsler, "Simulation and experimental validation of gaps and bridging in the automated fiber placement process," Science and Engineering of Composite Materials, p. 22: 1-18, 2013.

[2] D. H.-J. A. Lukaszewicz, C. Ward and K. D. Potter, "The engineering aspects of automated prepreg layup: History, present and future," Composites Part B: Engineering, p. 43: 997-1009, 2012.

[3] T. Wille, L. Kärger and R. Hein, "Adaptive, tolerant and efficient composite structures," in Adaptive, tolerant and efficient composite structures, Berlin, Heidelberg, Springer Berlin Heidelberg, 2013, pp. 199210.

[4] J. Llorca, C. Gonzalez, J. M. Molina-Aldareguia, J. Segurado, R. Seltzer, F. Sket, M. Rodriguez, S. Sadaba, R. Munoz and L. P. Canal, "Multiscale modeling of composite materials: A roadmap towards virtual testing," Advanced Materials, p. 23: 5130-5147, 2011.

[5] K. Fayazbakhsh, M. Nik, D. Pasini and L. Lessard, "Defect layer method to capture effect of gaps and overlaps in variable stiffness laminates made by Automated Fiber Placement," Composite Structures, pp. 245-251, 972013.

[6] J. Llorca, C. Gonzalez, J. M. Molina-Aldareguia and C. S. Lopes, "Multiscale Modeling of Composites: Toward Virtual Testing ... and Beyond," The Journal of The Minerals, Metals \& Materials Society, pp. 65: 215-225, 2013.

[7] M. R. Garnich and G. Karami, "Localized Fiber Waviness and Implications for Failure in Unidirectional Composites," Journal of Composite Materials, pp. 39:1225-1245, 2005. 
[8] P. D. Soden, M. J. Hinton and A. S. Kaddour, "Lamina properties, layup configurations and loading conditions for a range of fibre-reinforced composite laminates," Composites Sience and Technology, pp. 1011-1022, 581998.

[9] "Python Language Reference, version 2.7," Python Software Foundation, [Online]. Available: https://www.python.org/. [Accessed 0402 2016].

[10] K. Croft, L. Lessard and D. Pasini, "Experimental study of the effect of automated fiber placement induced defects on performance of composite laminates," Composites: Part A, pp. 42:484-491, 2011.

[11] L. Kärger and A. Kling, "As-built FE simulation of advanced fibre placement structures based on manufacturing data," Composite Structures, pp. 104-112, 2013.

[12] F. Heinecke, W. van den Brink and T. Wille, "Assessing the structural response of automated fibre placement composite structures with gaps and overlaps by means of numerical approaches," presented at the International Conference on Composite Materials, Copenhagen, 2015.

[13] R. Hill, "Elastic properties of reinforced solids: Some theoretical principles," Journal of the Mechanics and Physics of Solids, pp. 11:357-372, 1963.

[14] P. Suquet, "Elements of homogenization for inelastic solid mechanics," Homogenization techniques for composite media, pp. 193-278, 1985.

[15] “OpenTURNS," Électricité de France, EADS France, PHIMECA Engineering SA, [Online]. Available: http://www.openturns.org/. [Accessed 0402 2016].

[16] A. Manan and J. Cooper, "Design of Composite Wings Including Uncertainties: A Probabilistic Approach," Journal of Aircraft 46(2), pp. 601-607, 2009.

[17] A. Forrester, A. Sóbester and A. Keane, Engineering Design via Surrogate Modelling - A practical Guide, John Wiley Sons Ltd, 2008.

[18] A. Forrester and A. Kean, "Recent advances in surrogate-based optimization," Progress in Aerospace Sciences, pp. 50-79, 2009.

[19] A. Nik, K. Fayazbakhsh, D. Pasini and L. Lessard, "A comparative study of metamodeling methods for the design optimization of variable stiffness composites," Composite Structures, p. 494-501, 2014. 